Hacı Bekir Özerkan $\bowtie$

Can Çoğun

https://doi.org/10.21278/TOF.444007919

ISSN 1333-1124

eISSN 1849-1391

\title{
ELECTROCHEMICAL SMALL DIAMETER DEEP HOLE DRILLING OF POWDER METAL STEEL
}

\begin{abstract}
Summary
In this study, a new hybrid electrochemical drilling (ECD) method is proposed. The method makes use of a tubular tool capable of rotating and an electrolyte flushing through the inner hole at the same time. The designed and manufactured small size ECD machine's most important features are the regulation of the tool feed rate via current feedback control and the use of a rotating tubular tool with internal electrolyte flushing. The powder metal (PM) FLN2 4405 steel was drilled by using a brass tubular tool with various combinations of machining parameters such as machining voltage, electrolyte concentration and flushing pressure, and tool rotation speed. The machining performance outputs, namely, material removal rate (MRR), average radial overcut (RADOC), conicity (CO) and hole geometries were calculated and compared in different machining conditions. The results have shown that the aforementioned variables had a direct impact on the machining performance outputs in ECD. In all experiments, MRR increased with an in increase in machining voltage, tool rotational speed, electrolyte concentration and flushing pressure. RADOC values decreased with the rotational speed of the tool. The present study shows that deep holes with precise geometries and dimensions can be drilled successfully by employing the proposed hybrid ECD method. The method is economical and environmentally friendly since it requires low machining current values $(0.1$ to $0.7 \mathrm{~A})$ and a small amount of non-hazardous halide salt solutions instead of acidic solutions throughout the drilling process.
\end{abstract}

Key words: $\quad$ electrochemical drilling, rotational tubular tool, current feedback control, PM steel workpiece

\section{Introduction}

Most of the metals which exhibit high hardness, wear resistance, toughness and strain hardening properties cannot be machined easily and with desired dimensional accuracy, surface quality and geometries by employing traditional chip removal methods. Therefore, the use of unconventional hybrid manufacturing methods becomes inevitable in the machining of such metals. The electrochemical drilling (ECD) method is an attractive method and it is used in drilling of materials that are difficult to cut due to a lack of residual stresses, tool wear and burr, and limited shape deformation. The method makes use of usually salt solutions as electrolytes and gives a higher hole depth/diameter $(\mathrm{h} / \mathrm{d})$ ratio in the drilling of holes with relatively smaller diameters compared to conventional drilling operations [1-3]. In the ECD 
method, the cathode tool is separated from the anode workpiece by a small gap and the anode is dissolved at a rapid rate as a result of the applied electrical current to the electrolyte. In the classical ECD method, the removing of debris from the gap between the tool and the workpiece and the cooling of the medium during operation are achieved by pumping a high pressure electrolyte from the centre of the insulated tubular tools, made of lead, copper or stainless steel, towards the workpiece. The material dissolution from the workpiece occurs rapidly. Ionic residues and solid precipitates formed as a result of the electrolysis may clog the gap between the tool and the workpiece in deep drillings, which finally stops the drilling and causes the formation of shapeless holes [4]. Therefore, obtaining a desired hole depth and dimensional and geometrical hole accuracy are still the main concerns of the method [1-5]. In recent years, hybrid ECD methods have been developed to overcome the problems encountered in drilling with a high pressure acidic electrolyte.

Acidic drilling techniques, commonly known as electrochemical jet machining (ECJM), are classified into three groups. The first technique, which is called shaped tube electrochemical machining (STEM), utilizes insulated and acid resistant tubular electrodes [6-8]. Acids in 10$25 \%$ concentrations are the most preferred electrolytes in STEM as they lower machining voltages down to 5-15 V [2, 7, 9, 10]. Capillary drilling (CD) is the second ECJM technique and it is commonly preferred in the drilling of deep and small diameter holes [1-3, 9-11]. In this technique, an acidic electrolyte is fed through a tool containing a negatively polarized small diameter titanium wire in a tubular glass. The need for high drilling voltages (100-200 V) due to the non-conductive glass tube and the risk of glass tube breaking impose constraints on the technique. The third technique, called electro-stream drilling (ESD), is recommended for successful macro- and micro-scale drillings in which the electrolyte is polarized by a negatively charged platinum wire tool. The process is based on feeding the acidic electrolyte at the pressure of 0.3-1 MPa through a very small diameter capillary glass nozzle at 150-800 V [2, 912]. In the jet electrolytic drilling (JED) technique, which is developed in addition to ECJM techniques, the nozzle tip does not enter the hole during drilling; however, it is positioned with a stand-off distance of around $2-5 \mathrm{~mm}$. The drilling of a hole by using the JED process is achieved by the hitting of a high-pressure electrolyte (1-6 MPa) on the workpiece surface which causes anodic and acidic dissolution on the workpiece surface [6, 9-11, 13-16]. The technique necessitates high machining voltages $(400-800 \mathrm{~V})$ due to the positioning of the cathode tool at a distance above the workpiece surface. The targeted hole diameter depends on the nozzle hole diameter, current intensity, acid concentration and electrolyte fluid pressure. The main limitations of the technique are the hole tapering (around $5-10^{\circ}$ ) and the hole diameter expansion (the hole diameter can be four times higher than that of the jet). In recent years, a new technique, called laser jet electrochemical drilling (JECD-LD) method, is used to obtain more uniform hole shapes by adding laser energy to the process [17-19].

As mentioned above, there are many experimental and theoretical studies carried out on the hybridization of ECD by changing the tool shape, polarization, electrolyte type and electrolyte application methods, etc. In the here proposed new hybrid ECD method, the electrolyte is ejected at high pressure through a tubular rotary brass tool for drilling small diameter holes with a high $\mathrm{h} / \mathrm{d}$ ratio. There is a limited number of ECD studies in which the rotational movement is given to the tool [20-22]. In these studies, the lateral flushing has been applied to the machining gap; therefore, only shallow micro-holes with very small $\mathrm{h} / \mathrm{d}$ ratios were drilled in very thin metal plates. In this study, a small amount of electrolyte pool, which is sufficient for anodic dissolution, is formed by ejecting the electrolyte through the tool instead of the complete dipping of the tool and the workpiece into the electrolyte tank. Another important feature of the proposed hybrid ECD method is the control of the tool feed by current feedback signal. The investigation into the drilling performance in steel parts produced by powder metallurgy (PM) using the new proposed hybrid ECD technique is also considered as another distinguishing aspect of the present study. As it is known, finished 
surface, underlying microstructure, presence of pores, residual stresses and notches are the main effective features in the shaping and machining of PM steels and there is still plenty of room in the literature for defining machining parameters of the new generation of PM steels [23-26]. Therefore, in the present study, the drilling performance of the new hybrid ECD method was studied using PM steel plates. MRR, RADOC, $\mathrm{CO}$ and hole geometries were determined under different machining conditions using various combinations of machining voltage, tool rotational speed, concentration and flushing pressure of the electrolyte.

\section{Experimental procedures}

Figure 1 shows the ECD experimental setup used in the study. The mechanical body (1) was fabricated from cast iron due to its high vibration absorption capacity. The head of the workbench was able to travel along a vertical shaft by means of a square screw mechanism. A Maxon $\mathrm{A} / \mathrm{max} 15$ servo motor was used to rotate the tool electrode around the $\mathrm{z}$-axis. The servo motor was controlled by an MIP 10 driver control unit and the system made use of a PC program written in DELPHI. The required rotation speed for the precise feed control was given to the servo motor according to the measured current which passes through the tool and the workpiece. When the electrode came into contact with the workpiece, the electrode was automatically drawn back about $1 \mathrm{~mm}$. The machining gap was controlled by the machining current values measured continuously in milliseconds by a sensitive current sensor (2). An XRF 1200 model DC power supply (3) supplied machining current to the machining medium.

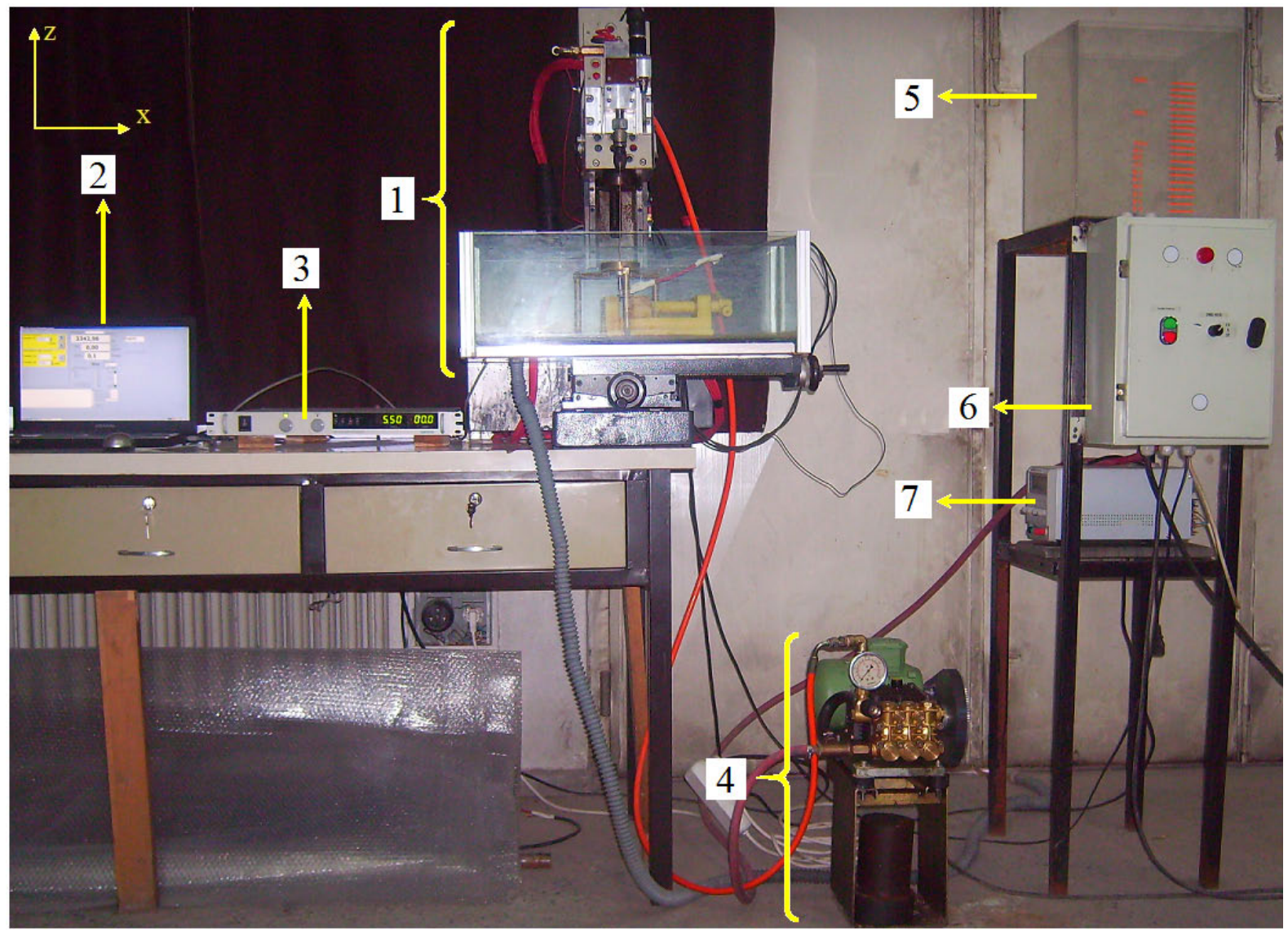

Fig. 1 The electrochemical drilling (ECD) unit; (1) ECD machine body, (2) computer (actuates the control software of tool motion and machining parameters), (3) DC power supply for machining current control, (4) high pressure pump and connection system, (5) electrolyte tank, (6) electronic control panel, (7) DC power supply for tool rotational speed control.

Four machining parameters, namely, machining voltage $(V)$, electrolyte concentration $(C)$, tool rotation speed $(n)$ and electrolyte flushing pressure $(P)$ were set to different values to 
conduct the experimental study (Table 1). The workpiece material was sinter hardened FLN2$4405 \mathrm{PM}$ steel rectangular in shape and with dimensions of $7 \times 50 \times 14 \mathrm{~mm}$. The chemical composition of the PM steel is given in Table 2. The as-received steel specimens had the hardness value of $18.5 \mathrm{HR}-\mathrm{C}$ and density of $7.4 \mathrm{~g} / \mathrm{cm}^{3}$. Two specimens were clamped together to obtain holes at the interface between the contacting surfaces (Figure 2). A hollow brass tube (for the inner hole electrolyte flushing) tool was used as electrode (Table 1). The tool electrode was mounted to the pressure head by means of a mandrel equipped with a sealing ring. The tool was supported by a ceramic guide to prevent lateral deflection (Figure 2). The sidewall of the electrode was insulated by $\mathrm{C}_{5} \mathrm{H}_{5} \mathrm{NO}_{2}$ cyanoacrylate coating that was $0.1 \mathrm{~mm}$ thick. $\mathrm{NaCl}$ solution, which is cheap and easy to find, was used as electrolyte instead of acidic solutions. The electrolyte was flushed through the tool hole by means of a pump placed into the electrolyte tank (Figure 2, (4)). In this study, the anodic removal took place in the electrolyte deposit (pool) ejected from the tool tip (Figure 2, (1)). $14 \mathrm{~mm}$ deep holes were also drilled using non-rotating tools to compare the drilling performance of the rotating tools (Table 1).

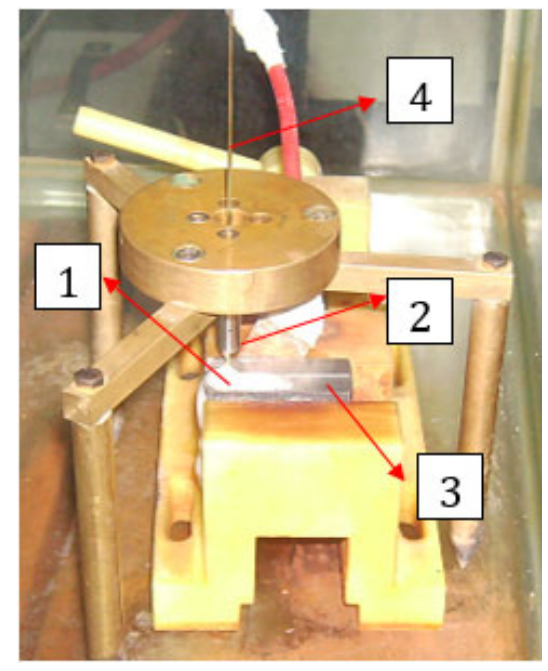

Fig. 2 Electrochemical drilling and working medium; (1) processing medium containing electrolyte deposit (pool), (2) ceramic tool guide, (3) PM workpiece pair (clamped), (4) brass tubular tool.

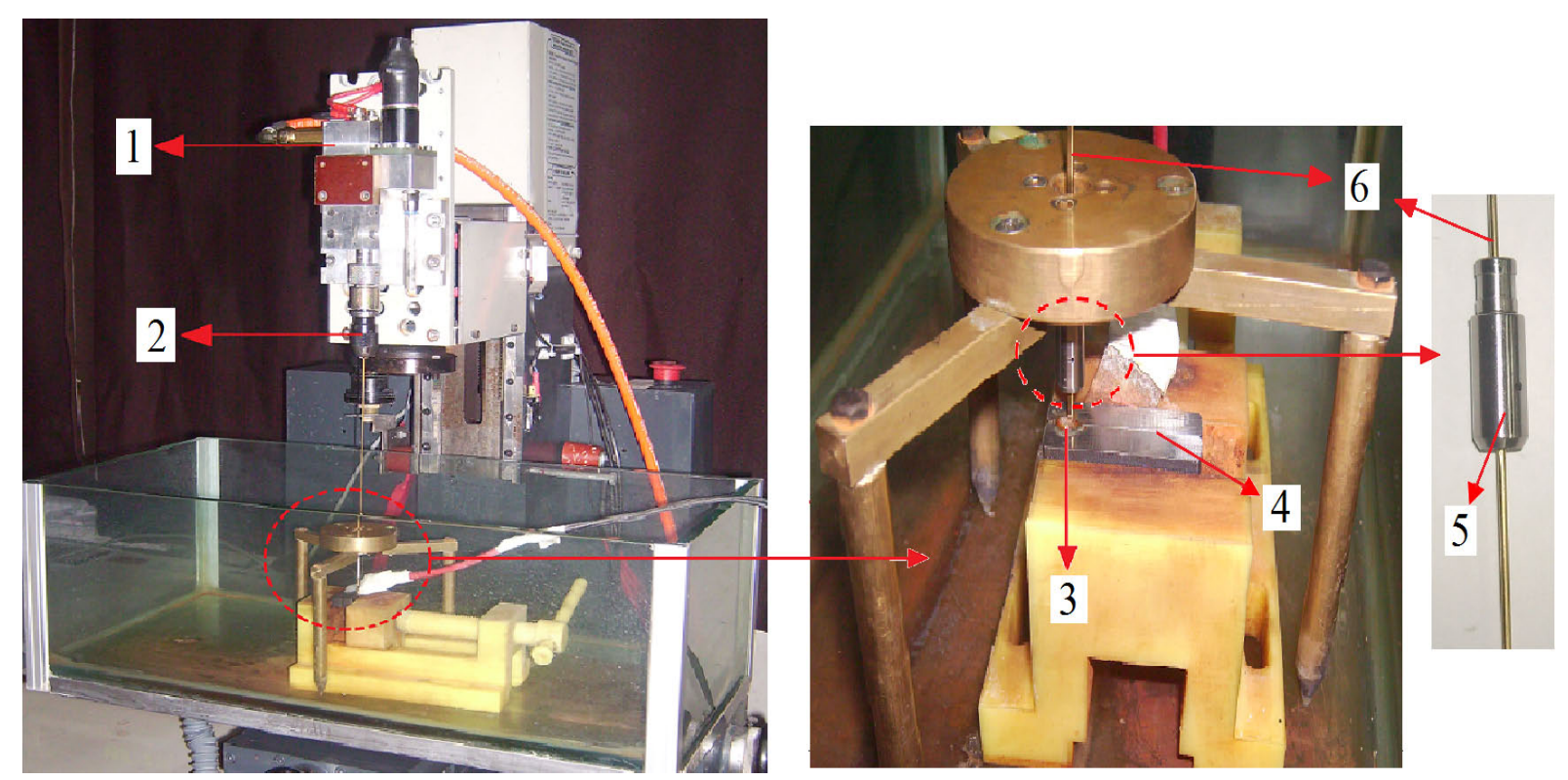

Fig. 3 Electrochemical drilling and working medium; (1) pressure head, (2) chuck linkage, (3) processing medium containing electrolyte deposit (pool), (4) PM workpiece pair (clamped), (5) ceramic tool guide, (6) brass tubular tool. 
Each drilling experiment was repeated three times and the average of the measurements were taken to ensure the reliability of the results. MRR (Eqn. 1) was determined by measuring the weights of the specimens before and after machining using a 0.001 gr sensitive digital precision balance. RADOC (Eqn. 2) and CO (Eqn. 3) were determined using the hole images obtained by a HP Photosmart C4280 high pixel (19200) scanner. The diameter of the holes was measured from images obtained in the scanned hole sections using image analysis and quantification programs (Datinf Measure). For each hole, at least ten measurements were taken to determine RADOC and $\mathrm{CO}$ values.

$$
\begin{aligned}
& \operatorname{MRR}\left[\frac{\mathrm{mm}^{3}}{\mathrm{~min}}\right]=\frac{\text { weight of the workpiece dissolved }}{\text { machining time } \times \text { workpiece density }} \\
& R A D O C[\mu m]=\frac{\text { average hole diameter }- \text { tool diameter }}{2} \\
& C O=\frac{\text { max. hole diameter }- \text { min. hole diameter }}{\text { hole depth }} \times 100
\end{aligned}
$$

Table 1 ECD machining parameters

\begin{tabular}{|c|c|}
\hline Parameters & Settings \\
\hline Workpiece & FLN2-4405 PM steel $(7 \times 50 \times 14 \mathrm{~mm})$ \\
\hline Tool & tubular brass, $D=0.5 \mathrm{~mm}, d=0.18 \mathrm{~mm}, L: 300 \mathrm{~mm}$ \\
\hline Drilling depth $[\mathrm{mm}]$ & 14 (through hole) \\
\hline Machining voltage $V,[$ Volt $]$ & $4.5-5.5-6.5$ \\
\hline Initial gap distance $[\mu \mathrm{m}]$ & 600 \\
\hline Electrolyte & NaCl solution \\
\hline Electrolyte concentration $C,[\mathrm{~g} / \mathrm{l}]$ & $100,125,150$ \\
\hline Electrolyte application & injection through tubular tool \\
\hline Injection pressure $P,[\mathrm{MPa}]$ & 2,4 \\
\hline Tool rotational speed $n,[\mathrm{rpm}]$ & $0,200,400$ \\
\hline Polarity & tool $(-)$, workpiece $(+)$ \\
\hline
\end{tabular}

Table 2 Chemical composition of FLN2-4405 PM steel according to the Metal Powder Industries Federation 35 standard

\begin{tabular}{|l|c|c|c|c|c|}
\hline & Fe [wt.\%] & C [wt.\%] & Ni [wt.\%] & Mo [wt.\%] & Mn [wt.\%] \\
\hline FLN2-4405 & Remaining & $0.4-0.7$ & $1.0-3.0$ & $0.65-0.95$ & $0.05-0.30$ \\
\hline
\end{tabular}




\section{Results and discussion}

Figure 4 shows some of the holes drilled with and without tool rotation. In both cases, the problem of clogging of the machining gap between the tool and the workpiece was not observed and the PM steel workpieces were easily dissolved during the drilling processes. Higher hole inlet diameters observed in some samples were caused by the delayed anodic dissolution of the workpiece in the electrolyte deposit (pool) (Figure 2, (1)) ejected from the tool tip at the beginning of the drilling.

As it is clearly seen in Figure 4, some holes with wavy wall surfaces and non-uniform geometries were obtained in drillings without tool rotation. Irregular hole geometries resemble those obtained by finite element modelling in the study of Wang and Zhu [27]. The presence of non-uniform hole geometries in non-rotating tool drillings is an undesirable situation. Local uneven anodic dissolution and gap clogging due to the machining sludge may lead to such non-uniform hole geometries. On the other hand, uniform hole geometries and smooth hole surfaces were obtained in drillings with tool rotation (Table 3). However, some of the holes drilled by rotational tools did not have perfectly uniform geometries and slight variations in the diameter were observed along the height of the holes (Figure 4, the 2nd, 4th and 9 th holes). Figure 5 displays the microstructure of the starting PM steel. Although the tool diameter used in the experiments was $500 \mu \mathrm{m}$, material inhomogeneity was observed in the figure in a region of the $800 \mu \mathrm{m}$ wide PM steel sample, as the average diameter of the resulting holes was about $800 \mu \mathrm{m}$. As can be seen, the sinter hardened steel contained micropores (dark regions) left from sintering and a mostly martensitic phase with some nickel rich regions (bright regions). The distribution of pores and phases was not homogenous in every part of the steel. Non-uniform geometries observed in the hole structures were attributed to the inhomogeneous distribution of pores, elements and phases. Because the electrochemical drilling caused the dissolution of structurally and compositionally different regions seen in Figure 5 at different rates, the sizes of the holes were not the same through the thickness of some steel specimens. It was believed that while drilling PM samples, the regions of sintered metal powders with different anodic dissolution properties caused variations in the hole diameters. Accordingly, coarse grained particles together with very small ionic residues might have blocked the lateral gap and reduced the current density locally in the regions which had different anodic dissolution properties. A decrease in the current density generally causes the slowing down of the feed rate, which finally results in the diameter expansion by dissolution of more material due to the slow tool feed motion. When tool passes over these non-uniform regions, the tool feed rate increases again as a result of an increase in the current density until its normal value is reached. Accordingly, the diameters of the holes change along the hole depth because of the variations in the current density during the operation. The authors believe that dissimilar dissolution behaviours of different regions of the PM workpiece materials, the size of the dissolution products and their flow behaviour in the gap were the main reasons for the current changes during the process.

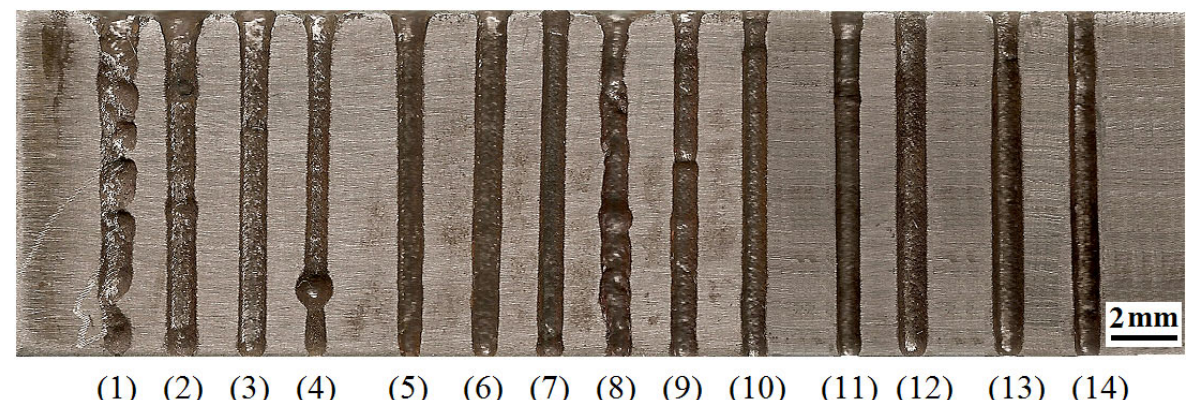

Fig. 4 Optical images of some holes drilled using non-rotational and rotational tools 
Table 3 Machining conditions of holes presented in Fig. 4

\begin{tabular}{|c|c|c|c|c|}
\hline Hole & $n[\mathrm{rpm}]$ & $P[\mathrm{MPa}]$ & $C[\mathrm{~g} / \mathrm{l}]$ & $V[$ Volt $]$ \\
\hline 1 & 0 & 2 & 100 & 4.5 \\
\hline 2 & 200 & 2 & 100 & 4.5 \\
\hline 3 & 400 & 2 & 100 & 4.5 \\
\hline 4 & 400 & 2 & 100 & 4.5 \\
\hline 5 & 0 & 4 & 100 & 4.5 \\
\hline 6 & 200 & 4 & 100 & 4.5 \\
\hline 7 & 400 & 4 & 100 & 4.5 \\
\hline 8 & 0 & 2 & 125 & 4.5 \\
\hline 9 & 200 & 2 & 125 & 4.5 \\
\hline 10 & 400 & 2 & 125 & 4.5 \\
\hline 11 & 200 & 2 & 100 & 5.5 \\
\hline 12 & 400 & 2 & 100 & 5.5 \\
\hline 13 & 200 & 4 & 100 & 5.5 \\
\hline 14 & 400 & 4 & 100 & 5.5 \\
\hline
\end{tabular}

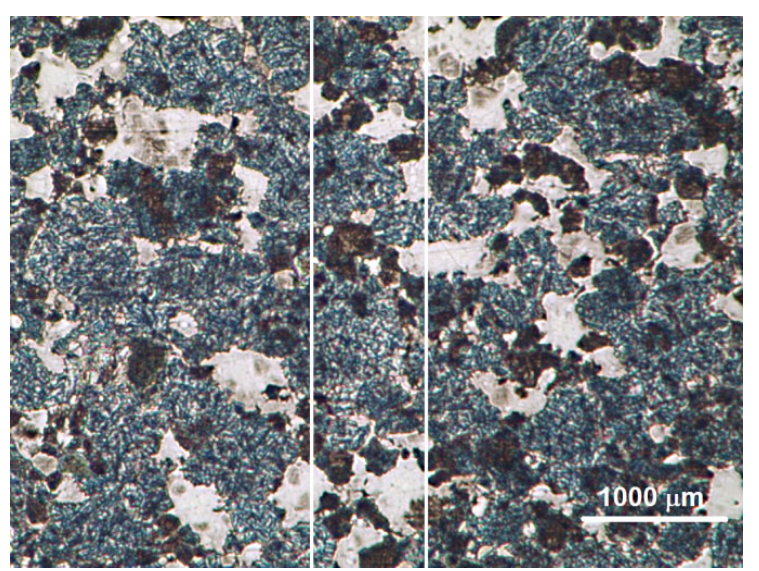

Fig. 5 Microstructure of a PM steel specimen. Two parallel lines indicate a part of a hole $800 \mu \mathrm{m}$ in diameter.

Drilling performance is highly influenced by machining voltage $(V)$ and electrolyte concentration $(C)$. The increase in $V$ and $C$ results in faster drillings because of the easy removal of the material and the debris from the processing environment as it was observed in the present study. Figure 6 shows the variation of MRR with $V$ for various machining settings of $C$, tool rotational speed $(n)$, and flushing pressure $(P)$ of the electrolyte. It is clearly seen that an increase in $P$ increased the MRR significantly. Possibly, the application of a higher $P$ enabled easy removal of debris from the processing gap. Thus, the waiting time for the dissolution of metal ions in the electrolyte was shortened and the drilling was stabilized. That is to say, the $V$ value did not change for a long time and the current density was set according to the selected $C$ value. Moreover, a variation in the current value was not observed with the increase in $P$. Figure 6 also emphasizes the importance of $n$ for the drilling performance. MRR values displayed an apparent increase with the increase in $n$. The combination of higher $\mathrm{n}$ and $P$ values resulted in the more effective removal of the electrolyte containing dissolved material from the front and lateral machining gaps. Therefore, conductivity of the machining gap was preserved during the process and the MRR was enhanced. On the other hand, drilling with non-rotational tool caused lower MRR values (longer processing times) compared to drillings with rotation. Due to the high electrochemical dissolution capability of the PM material, non-rotational tools were also able to drill a hole; however, the hole quality was 
poor. The highest MRR values for rotary and stationary tools were found to be $1.19 \mathrm{~mm}^{3} / \mathrm{min}$ at $6.5 \mathrm{~V}, 400 \mathrm{rpm}, 150 \mathrm{~g} / \mathrm{l}, 4 \mathrm{MPa}$ settings (Figure $6 \mathrm{f}$ ) and $0.406 \mathrm{~mm}^{3} / \mathrm{min}$ at $4.5 \mathrm{~V}, 100 \mathrm{~g} / \mathrm{l}$, $2 \mathrm{MPa}$ settings (Figure 6a), respectively. The experimentally obtained values indicated that the rotation of the tool electrodes improved the MRR between $14 \%(4.5 \mathrm{~V}, 2 \mathrm{MPa}, 125 \mathrm{~g} / \mathrm{l})$ and $47 \%(5.5 \mathrm{~V}, 2 \mathrm{MPa}, 150 \mathrm{~g} / \mathrm{l})$ for different machining settings. In the case of the use of the rotational tool, the consumption of the electrolyte $(200-700 \mathrm{ml} / \mathrm{hole})$ was less than in the case of the use of the non-rotational tool (900-2000 $\mathrm{ml} / \mathrm{hole})$ since the hole was drilled in a shorter time.

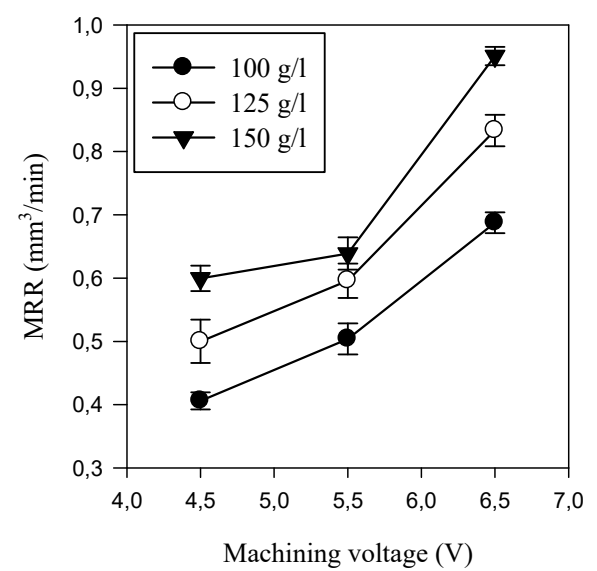

(a)

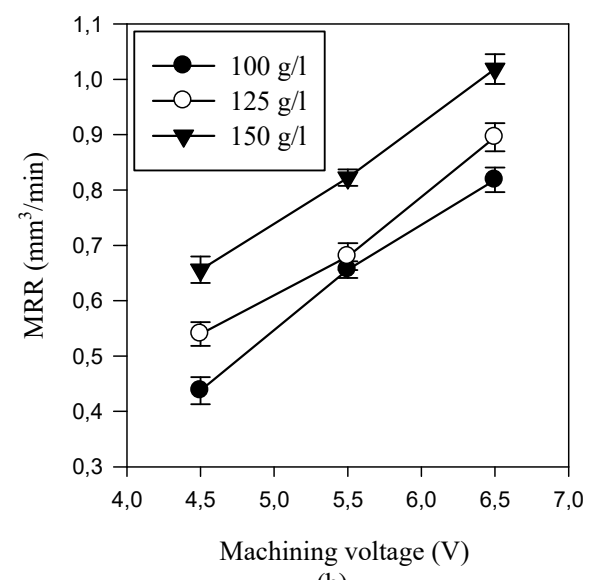

(b)

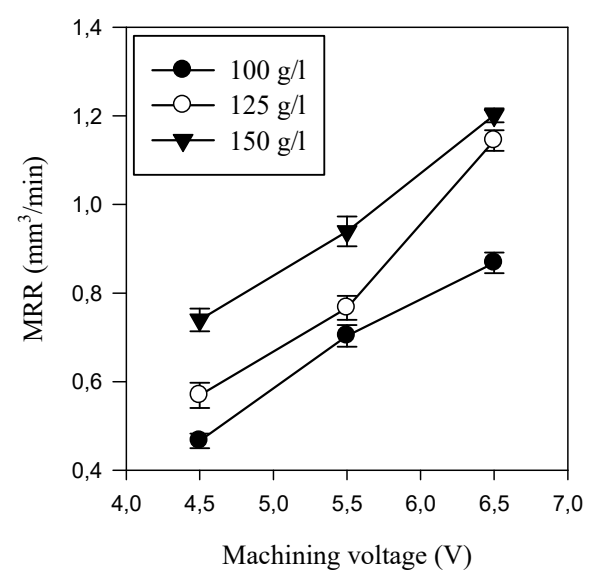

(c)

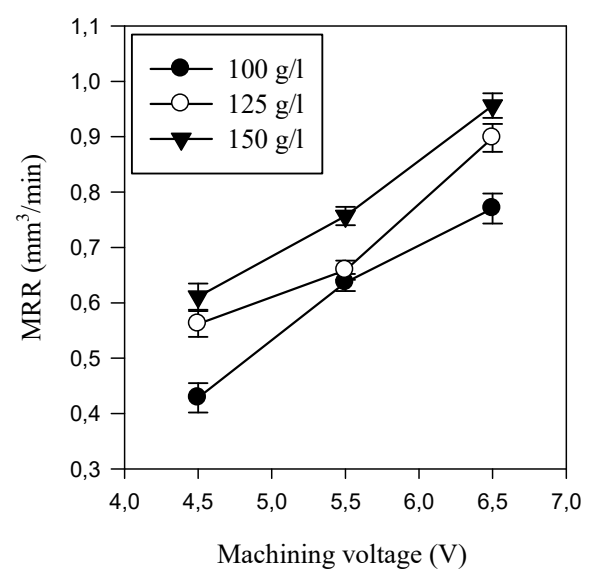

(d)

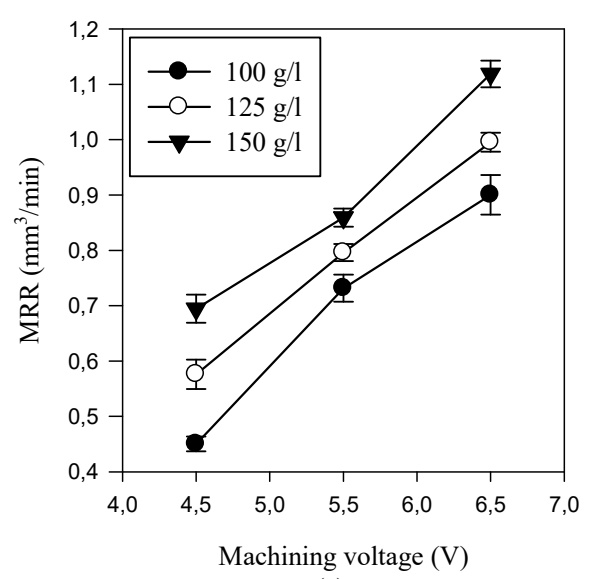

(e)

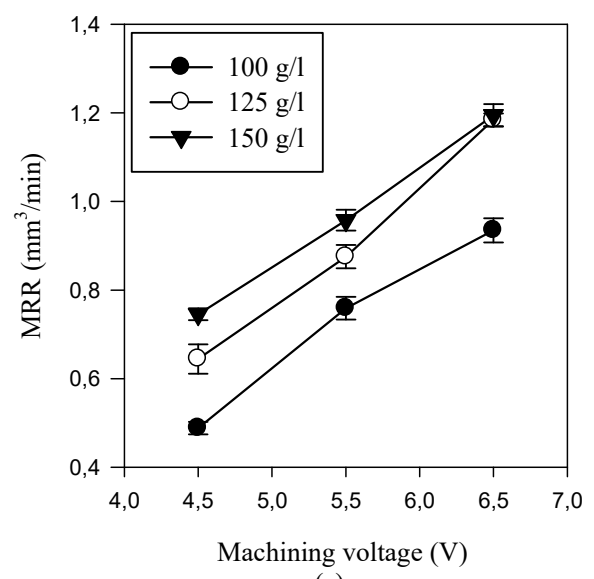

(e)

Fig. 6 Variation of MRR with different machining settings. (a) Non-rotating tool, $2 \mathrm{MPa}$, (b) $200 \mathrm{rpm}, 2 \mathrm{MPa}$, (c) $400 \mathrm{rpm}, 2 \mathrm{MPa}$, (d) non-rotating tool, $4 \mathrm{MPa}$, (e) $200 \mathrm{rpm}, 4 \mathrm{MPa}$, (f) $400 \mathrm{rpm}$, $4 \mathrm{MPa}$. 


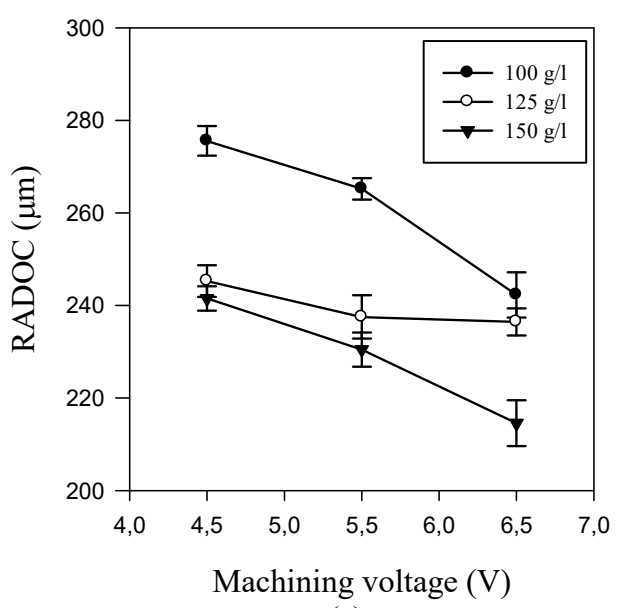

(a)

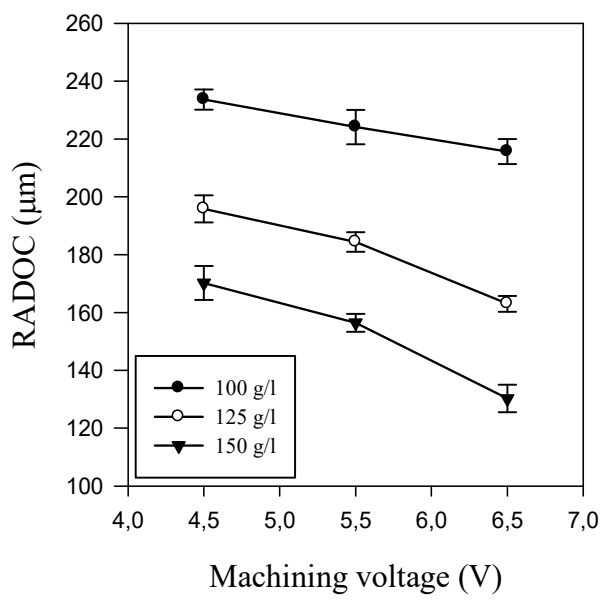

(b)

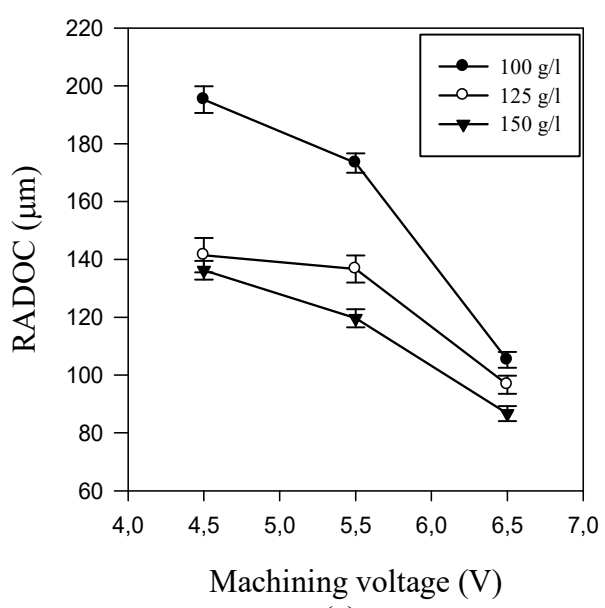

(c)

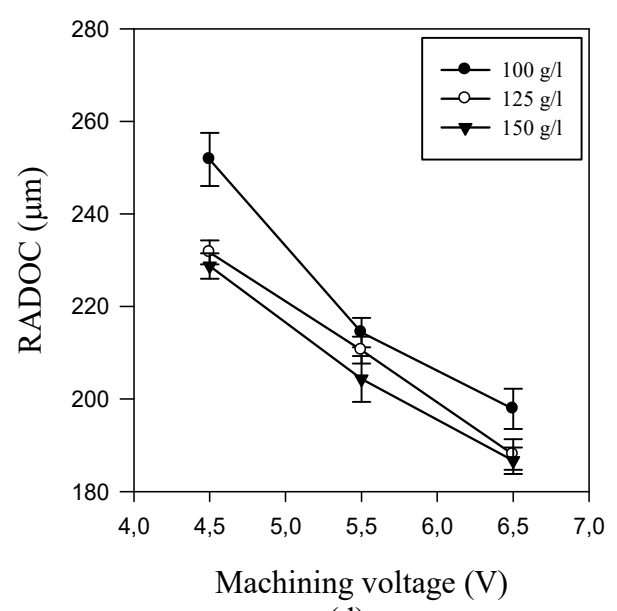

(d)

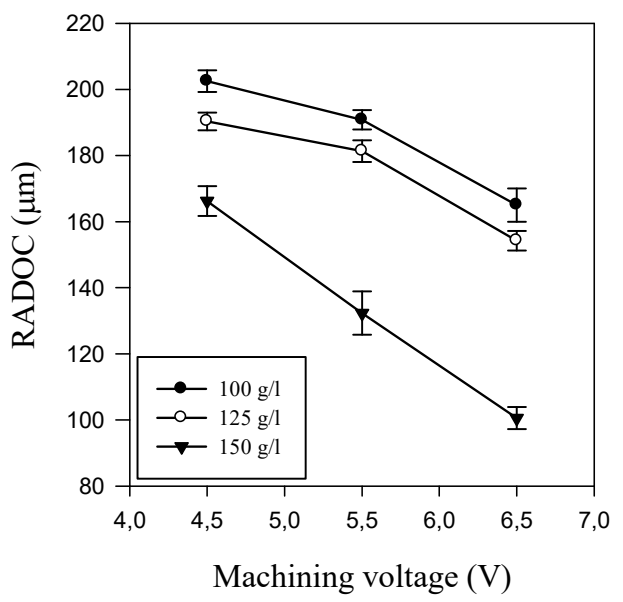

(e)

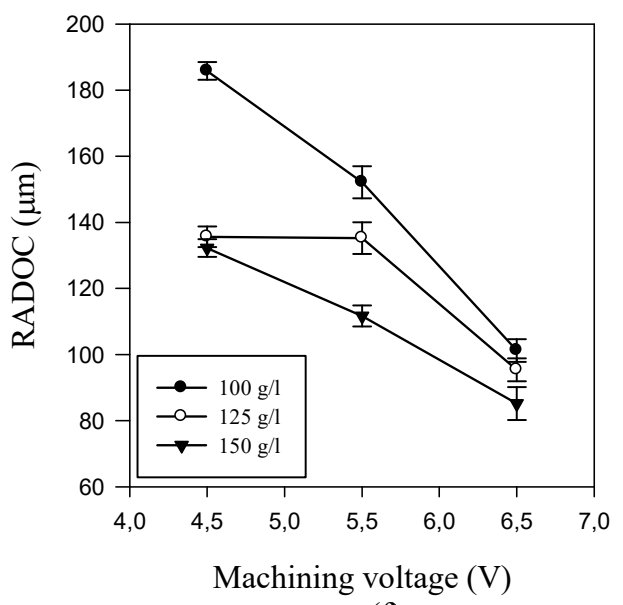

(f)

Fig. 7 Variation of RADOC with different machining settings. (a) Non-rotating tool, $2 \mathrm{MPa}$, (b) $200 \mathrm{rpm}$, $2 \mathrm{MPa}$, (c) 400rpm, $2 \mathrm{MPa}$, (d) non-rotating tool, $4 \mathrm{MPa}$, (e) $200 \mathrm{rpm}, 4 \mathrm{MPa}$, (f) $400 \mathrm{rpm}, 4 \mathrm{MPa}$.

It is clearly seen in Figure 7 that the RADOC values were reduced when $V, C$ and $n$ increased. The increase in $\mathrm{NaCl}$ concentration makes the lateral and frontal gaps more conductive, thereby shortening the gap distance and increasing the current density. As a result, durations of drilling were shortened and the average lateral overcut values decreased. 
The PM material containing sintered powders displayed easy anodic dissolution behaviour and the machining sludges were small enough to be easily swept away from the lateral gap by means of high electrolyte pressure and tool rotation. As shown in Figure 7, the RADOC values decreased with an increase in $P$ in the case of drillings without and with tool rotation. Moreover, the increase in $n$ also led to a decrease in RADOC. Nevertheless, the larger hole diameters, the wavy hole surfaces with non-uniform hole geometries and the longer drilling durations were observed in the non-rotational tool drillings. The increase in $V$ led to a decrease in RADOC in all experiments. The lowest RADOC value was $71.6 \mu \mathrm{m}$ at $6.5 \mathrm{~V}, 400 \mathrm{rpm}, 150 \mathrm{~g} / \mathrm{l}$, and $4 \mathrm{MPa}$ settings (Figure 7f), while the maximum value, found in the drilling with the non-rotating tool, was $275.6 \mu \mathrm{m}$ at $4.5 \mathrm{~V}, 100 \mathrm{~g} / \mathrm{l}$ and $2 \mathrm{MPa}$ settings (Figure 7a).

Higher $\mathrm{CO}$ values were obtained in the non-rotational tool drillings (Figure 4). The minimum and maximum $\mathrm{CO}$ values in the experiments were calculated as $0.49 \%$ and $6.86 \%$, respectively. Higher $P, C$ and $n$ yielded lower $\mathrm{CO}$ values in the drilling of PM steels.

\section{Conclusion}

An experimental ECD device was designed, fabricated and implemented in the PM steel drilling of deep holes with small diameters. The distinguishing features of the study are: (i) the introduction of a rotary tubular tool electrode with through-hole electrolyte flushing, (ii) the control of the tool's down feed by a current feedback signal and (iii) the use of a PM steel workpiece specimen, which adversely affects the hole geometry due to its non-homogenous microstructure and composition distribution. An extensive experimental study on the effects of various machining parameters such as material removal rate (MRR), radial overcut (RADOC) and conicity (CO) was carried out using the fabricated ECD device. The important findings of the study are as follows:

- Sinter hardened PM steel demonstrated good anodic dissolution behaviour with the application of tool rotation and high electrolyte pressure. Finer hole geometries and smoother hole surfaces were obtained and the gap clogging problem, typically observed in non-rotating tool drillings, was completely eliminated. The rotation of the electrode also improved he removal of the debris in the front and lateral gap between the tool and the workpiece.

- The tool/workpiece pair was not completely wetted with the electrolyte. Anodic dissolution took place in the electrolyte deposit (pool) ejected from the tool. Therefore, a small amount of electrolyte (maximum $700 \mathrm{ml}$ for the rotating tool and $2000 \mathrm{ml}$ for the non-rotating tool) was applied throughout the drilling process.

- The debris in the lateral gap was effectively swept away. Therefore, a higher current density and feed rate were obtained. Additionally, the hole diameter expansion was lowered (i.e. lower RADOC) at high tool rotational speeds $(n)$. The largest RADOC and $\mathrm{CO}$ values were found in the non-rotating tool drillings. The minimum and maximum $\mathrm{CO}$ values were $0.49 \%$ and $6.86 \%$, and high $P, C$ and $n$ settings were found to decrease $\mathrm{CO}$ values.

- The MRR became higher with an increase in $V, P$ and $n$ in all conducted experiments.

- No wear and spark craters were observed in the experiments on the tools, confirming the proper functioning of the tool feed control circuit and software.

- The proposed method can be considered as an economical and environmentally friendly method since it requires low current values $(0.1$ to $0.7 \mathrm{~A})$ and a small amount of non-hazardous halide salt solutions in the ECD of holes. 


\section{REFERENCES}

[1] M. Sen, H. Shan, A review of electrochemical macro-to micro-hole drilling processes, International Journal of Machine Tools and Manufacture, 45(2) (2005) 137-152. https://doi.org/10.1016/j.ijmachtools.2004.08.005

[2] K. Kumar, D. Zindani, J. P. Davim, Electrochemical Processes. In Advanced Machining and Manufacturing Processes Springer, (2018).

[3] H. A. Youssef, Machining of stainless steels and super alloys: traditional and nontraditional techniques, John Wiley \& Sons, New York, (2015). https://doi.org/10.1002/9781118919514

[4] S. Sharma, V. Jain, R. Shekhar, Electrochemical drilling of inconel superalloy with acidified sodium chloride electrolyte. The International Journal of Advanced Manufacturing Technology, 19 (7) (2002) 492-500. https://doi.org/10.1007/s001700200052

[5] D. S. Bilgi, V. Jain, R. Shekhar, S. Mehrotra, Electrochemical deep hole drilling in super alloy for turbine application. Journal of materials processing technology, 149 (1-3) (2004) 445-452. https://doi.org/10.1016/j.jmatprotec.2003.09.008

[6] J. Kozak, K. Rajurkar, R. Balkrishna, Study of electrochemical jet machining process, Journal of Manufacturing Science and Engineering, 118 (4) (1996) 490-498. https://doi.org/10.1115/1.2831058

[7] Z. Li, X. Wei, J. Sun, C. Zang, C. Fu, Y. Guo, Process capability and effect size of vacuum extraction shaped tube electrolytic drilling of Inconel alloy for high-performance cooling hole, The International Journal of Advanced Manufacturing Technology, 85 (9-12) (2016) 2557-2566. DOI 10.1007/s00170-0158085-z_ https://doi.org/10.1007/s00170-015-8085-z

[8] A. K. De Silva, J. R. Gamage, C. S. Harrison, Assessment of environmental performance of shaped tube electrolytic machining (STEM) and capillary drilling (CD) of superalloys. CIRP Annals, 66 (1) (2017) 57-60. https://doi.org/10.1016/j.cirp.2017.04.110

[9] G. Benedict, Nontraditional manufacturing processes, Routledge, New York, USA, (2017).

[10] H. A.-G. El-Hofy, Advanced machining processes: nontraditional and hybrid machining processes, McGraw Hill Professional, New York, USA, (2005).

[11] M. Sen, H. Shan, Analysis of hole quality characteristics in the electro jet drilling process, International Journal of Machine Tools and Manufacture, $45 \quad$ (15) (2005) 1706-1716. https://doi.org/10.1016/j.ijmachtools.2005.03.005

[12] M. R. Mahbub, M.P. Jahan, Micromachining of Titanium Alloys. In Micro and Nano Machining of Engineering Materials, Springer, (2019).

[13] M. Sen, H. Shan, Electro jet drilling using hybrid NNGA approach, Robotics and Computer-Integrated Manufacturing, 23 (1) (2007) 17-24. https://doi.org/10.1016/j.rcim.2005.08.004

[14] N. Jain, A. Potpelwar, S. Pathak, N. Mehta, Investigations on geometry and productivity of micro-holes in Incoloy 800 by pulsed electrolytic jet drilling, The International Journal of Advanced Manufacturing Technology, 85 (9-12) (2016) 2083-2095. https://doi.org/10.1007/s00170-016-8342-9

[15] A. Speidel, J. Mitchell-Smith, D. A. Walsh, M. Hirsch, A. Clare, Electrolyte jet machining of titanium alloys using novel electrolyte solutions, Procedia CIRP, $42 \quad$ (2016) 367-372. https://doi.org/10.1016/j.procir.2016.02.200

[16] H. Goel, P.M. Pandey, Experimental investigations into the ultrasonic assisted jet electrochemical microdrilling process. Materials and Manufacturing Processes, 32 (13) (2017) 1547-1556. https://doi.org/10.1080/10426914.2017.1279294

[17] M.S. Amalnik, J. McGeough, Intelligent concurrent manufacturability evaluation of design for electrochemical machining, Journal of materials processing technology, 61 (1-2) (1996) 130-139. https://doi.org/10.1016/0924-0136(96)02477-6

[18] S. H. Ahn, S. H. Ryu, D. K. Choi, C. N. Chu, Electro-chemical micro drilling using ultra short pulses, Precision Engineering, 28 (2) (2004) 129-134. https://doi.org/10.1016/j.precisioneng.2003.07.004

[19] A. Malik, A. Manna, Study on precision microholes using pulsed laser with jet electrochemical machining, Arabian Journal for Science and Engineering, 43 (9) (2018) 4593-4608. https://doi.org/10.1007/s13369-017-2998-9

[20] W. Zhao, X. Li, Z. Wang, Study on micro electrochemical machining at micro to meso-scale, In 2006 1st IEEE International Conference on Nano/Micro Engineered and Molecular Systems, (2006) 325-329. https://doi.org/10.1109/NEMS.2006.334733 
[21] H. P. Tsui, J. C. Hung, J. C. You, B. H. Yan, Improvement of Electrochemical Microdrilling Accuracy using Helical Tool, Materials and Manufacturing Processes, 23 (5) (2008) 499-505. https://doi.org/10.1080/10426910802104237

[22] T. Sekar, R. Marappan, Improving Material Removal Rate of Electrochemical Machining by Using Rotating Tool, Manufacturing Engineering, 8 (2) (2008) 60-63.

[23] M. Moravej, F. Chagnon, P. Francois, Machinability of Sinter-hardening PM materials with experimental machining enhancers, Transactions of Powder Metallurgy Association of India, 41 (1) (2015) 26-32.

[24] Bouzakis, K.D., König, W., Vossen, K. (1982). Use of Powder Metallurgical High Speed Steel in Gear Hobbing and Gear Shaping. CIRP Annals-Manufacturing Technology, 31(1), 25-29. https://doi.org/10.1016/S0007-8506(07)63262-4

[25] A. Klink, Y. Guo, F. Klocke, Surface integrity evolution of powder metallurgical tool steel by main cut and finishing trim cuts in wire-EDM, Procedia Engineering, 19 (2011) 178-183. https://doi.org/10.1016/j.proeng.2011.11.098

[26] A. Šalak, M. Selecká, H. Danninger, Machinability of powder metallurgy steels, Cambridge Int. Science Publishing (2005).

[27] M. Wang, D. Zhu, Simulation of fabrication for gas turbine blade turbulated cooling hole in ECM based on FEM, Journal of materials processing technology, 209 (4) (2009) 1747-1751. https://doi.org/10.1016/j.jmatprotec.2008.04.035

Submitted: $\quad 20.8 .2019$.

Accepted:

16.5.2020
Dr. Hacı Bekir ÖZERKAN*

Department of Machinery and Metal

Technologies,

Gazi University, Ankara, 06374, Turkey

Prof. Dr. Can ÇOĞUN

Mechatronics Engineering Department

Faculty of Engineering,

Çankaya University, Ankara, Turkey

*ozerkan@gazi.edu.tr 